\title{
LE DOSAGE DES PROTÉINES DU LAIT A L'AIDE DE LA RÉACTION XANTHOPROTÉIQUE
}

\author{
par
}

\section{M. BURUIANA}

Nous avons proposé autrefois la réaction xanthoprotéique pour le dosage colorimétrique des protéines du lait [2], du sang [3] et de l'urine [4].

Plus tard Lefaux [5] a utilisé aussi la même réaction, pour le dosage des polypeptides du sang.

Parmi les autres réactions colorées, la réaction du biuret, proposée pour la première fois dans le même, but par le savant roumain Em. Riegler [6], est la plus employée, avec ses nombreuses macro et micro-variantes.

A cause de la simplicité et de sa rapidité d'exécution, nous avons cherché à accroître aussi la sensibilité et l'exactitude de notre méthode.

Dans le présent travail nous exposons la nouvelle technique et quelques observations relatives aux facteurs qui influencent l'intensité de la réaction xanthoprotéique.

Les principales critiques qu'on a apportées à l'ancienne technique se référent à l'instabilité de la solution étalon à l'irrégularité de la réaction de nitration des protéines [1], et à son exécution assez difficile dans la pratique courante de la laiterie [7].

Pour éluder ces critiques nous avons substitué à l'étalon minéral l'appréciation photocolorimétrique. En l'absence du photocolorimètre, on peut recourir au colorimètre de Duboseq, utilisant comme étalon une solution chlorhydrique (m-100) de caséine, dont la conservation a été assurée par addition de formaldéhyde $(2 \%)$ et dont la concentration exacte est connue (Kjeldahl). Quant à la nitration des protéines, on a constaté qu'elle dépendait : a) de la concentration en acide nitrique, $b$ ) de la température de chauffage, et $c$ ) de la durée du chauffage.

Pour obtenir des résultats reproductibles, il faut travailler dans des conditions bien précisées qui sont les suivantes : la quantité de protéine à nitrer ne doit pas dépasser 6 milligrammes pour 1 millilitres d'acide nitrique de $\mathrm{D}=1,40$ (conc. $65,30 \%$ ) ; le volume de la solution protéique sera au maximum demi de celui de l'acide nitrique utilisé. La température de nitration doit dépasser $100^{\circ}$. C'est pour cela qu'il faut chauffer directement à la flamme. Pour plus de régularité, on peut employer un bain de glycérine qui bout à $120^{\circ}$.

La durée du chauffage ne doit pas dépasser $30-40$ secondes. 
Le chauffage prolongé risque d'oxyder et de décomposer la protéine.

Après nitration et refroidissement, on neutralise à la soude, en ajoutant un léger excès pour assurer un $p \mathrm{H}>9$, valeur pour laquelle l'intensité du couleur est maxiumm.

Toutes ces conditions sont réalisées dans la technique suivante, établie pour le dosage de diverses protéines du lait.

\section{I. - Détermination des protéines totales du lait}

On dilue le lait en proportion de 1,5 ou 1:10, d'après sa concentration en protéine. Dans une éprouvette de Iéna de $18 \times 180$ millimètres on met jusqu'à $0 \mathrm{ml}$. 5 du lait ainsi dilué, et on ajoute 1 millilitre d'acide nitrique $(\mathrm{D}=1,40)$. On chauffe directement sur flamme jusqu'à ébullition en continuant encore 20-30 secondes. Après refroidissement, on neutralise avec environ $1 \mathrm{ml} .6$ de soude à $40 \%$. Pour assurer un léger excès, après le virage de la couleur, on ajoute encore 1-2 gouttes. L'intensité du couleur atteint son maximum immédiatement et reste constante pendant plus de 24 heures. On complète à 10 millilitres avec de l'eau et l'on filtre sur papier en retournant le liquide jusqu'à parfaite limpidité. On lit ensuite l'extinction à l'écran bleu ( $\mathrm{L}=430 \mathrm{~m})$, dans les cuves de 1 centimètre du photocolorimètre. A l'aide de la courbe étalon, on déduit la quantité de protéine et on rapporte à 100 millilitres lait.

\section{II. - Dosage de la caséine}

Dans une fiole à centrifugation en verre d'Iéna de 12 millilitres, on met la même quantité de lait qu'auparavant. On ajoute 3 millilitres de mélange tampon (acide acétique $\mathrm{m} / 10$ et acétate de sodium $\mathrm{m} / 10 \mathrm{l}: 1, p \mathrm{H}=4,7)$ pour précipiter la caséine. Après centrifugation, on lave le précipité 2 fois chacune à 3 millilitres du même mélange tampon et après égouttage on ajoute sur le précipité 1 millilitre d'acide nitrique $(D=1,40)$. On procède comme plus haut dans la fiole même.

La différence entre les protéines totales et la caséine représente les protéines du sérum.

\section{III. - Dosage direct des protéines du sérum}

Dans une fiole de centrifugation, on met 1 millilitre de lait non dilué. On précipite la caséine avec 9 millilitres de tampon acétique de $p \mathrm{H}=4,7$. Après agitation, on centrifuge et on transvase 5 millilitres du liquide surnageant dans une autre fiole. Les protéines du sérum sont précipitées à chaud avec une volume égal d'acide trichloracétique à $10 \%$. On sépare le précipité par centrifugation et, ensuite, on lave 2 fois à l'acide trichloracétique à $5 \%$. Après égout- 
tage, on ajoute 1 millilitre d'acide nitrique et on procède comme de coutume.

\section{IV. - Etalonnage de la courbe photocolorimétrique}

Pour tracer la courbe extinction : concentration, on procède comme suit :

On précipite à chaud les protéines d'un millilitre de lait avec 9 millilitres d'acide trichloracétique à $10 \%$. Après filtration et lavage, on tranvase le filtre avec le précipité dans un matras Kjeldahl en déterminant, après minéralisation, la quantité de protéines.

Par ailleurs, on lit au photocolorimètre l'extinction des couleurs obtenues en procédant comme au point I, avec les quantités : 0,2-0,1-0,05-0,02 millilitres du même lait.

\section{Exemple :}

Protéines totales du lait (au Kjeldahl) $\ldots \ldots \ldots \ldots \ldots \ldots \ldots 2,65$ gr. \% Extinction avec 0,2 $\mathrm{ml}$. de lait $=1,18$ contenu en protéine 5,5 $\mathrm{mgr}$. \% Extinction avec 0,1 ml. de lait $=0,60$ contenu en protéine 2,65 mgr. \% Extinction avec $0,05 \mathrm{ml}$. de lait $=0,30$ contenu en protéine $1,325 \mathrm{mgr}$. \% Extinction avec 0,02 ml. de lait $=0,125$ contenu en protéine 0,53 mgr. \%

Dans le système d'axes rectangulaires unissant les points représentant l'extinction pour la concentration respective en protéines, on obtient une droite passant par l'origine. On remarque (fig. 1)

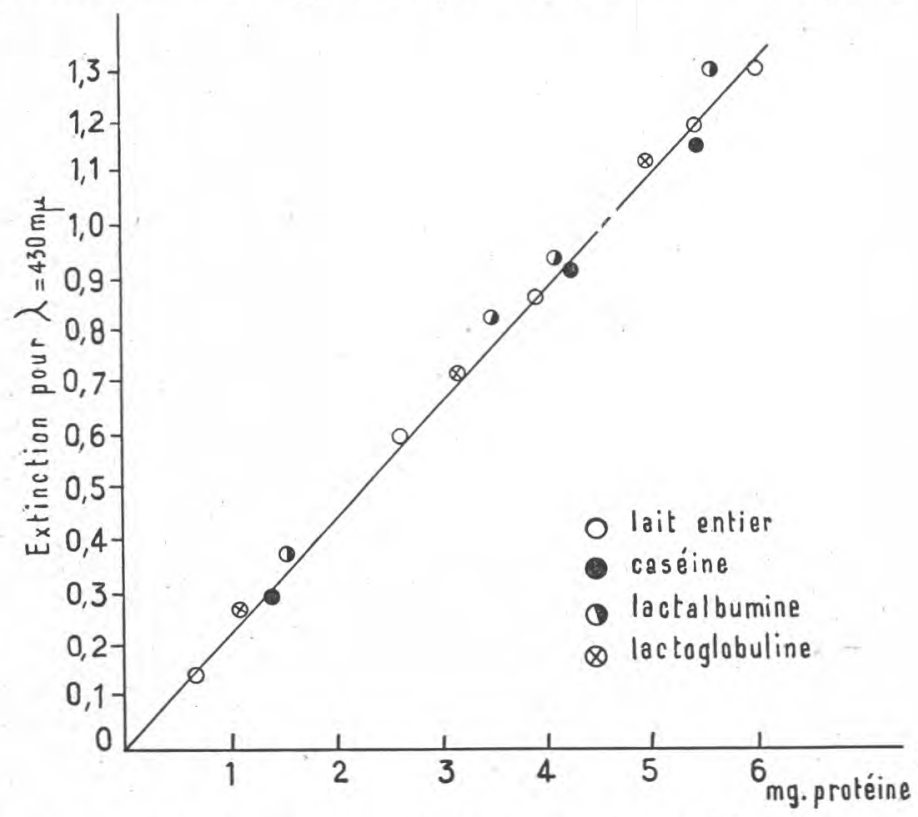

Fig. 1. - Variation de l'intensité de la réaction xanthoprotéique avec la concentration de diverses protéines du lait. 
qu'il existe une concordance rigoureuse entre la concentration en protéine et l'extinction.

On doit mentionner que la pente de la droite n'est pas la même pour toutes les protéines. Dans le cas du lait, nous avons toutefois constaté que la différence était très petite, l'extinction équivalente (1) ayant presque la même valeur pour toutes les catégories de protéines. Par contre, l'extinction équivalente des protéines d'autre origine varie largement. Cette question fera l'objet d'un autre Mémoire.

En général, on tient le noyau de la tyrosine comme le plus susceptible de nitration. On évalue pour cela en tyrosine l'intensité de la couleur obtenue après la nitration d'une protéine, en désignant même cette valeur par l'expression "indice de tyrosine ".

Il résulte du tableau suivant qu'il n'existe cependant aucun rapport entre le contenu en tyrosine d'une protéine et l'intensité de la couleur obtenue dans le cas des protéines du lait.

GONTENU EN TYROSINE DE QUELQUES PROTEINES DU LAIT

\begin{tabular}{|c|c|c|c|}
\hline Protéines & $\begin{array}{c}\text { Contenu } \\
\text { en tyrosine } \\
\%\end{array}$ & $\begin{array}{c}\text { Extinction } \\
\text { équivalente } \\
\text { (extinction pour } \\
1 \text { mgr, de protéine) }\end{array}$ & $\begin{array}{c}\text { Contenu en } \\
\text { tyrosine \% } \\
\text { correspondant à } \\
\text { l'extinction } \\
\text { équivalente }\end{array}$ \\
\hline Caséine ........ & $4,16(2)$ & 0,225 & 10,85 \\
\hline Lactoglobuline: . & $2,65(3)$ & 0,23 & 11,25 \\
\hline Lactalbumine .. & $0,95(4)$ & 0,24 & 11,50 \\
\hline
\end{tabular}

Le fait a été vérifié aussi par nous dans le cas d'autres protéines. Il est fort probable que non seulement la composition chimique mais aussi l'arrangement des molécules dans l'édifice macromoléculaire contribue à l'intensité de la couleur.

(1) Extinction pour 1 milligramme de protéine.

(2) Le contenu en tyrosine de la caséine a été déterminé par nous. Dans la littérature on trouve des chiffres oscillant entre : $3,9 \%(T$. Osborne) et $6,7 \%$ (B. I. Zbarsky).

(3) On désigne par lactoglobuline, la fraction du sérum acétique du lait précipité à $50 \%$ de saturation avec le sulfate d'ammonium. Le contenu en tyrosine a été déterminé par nous.

(4) On désigne par lactalbumine, la fraction du sérum acétique du lait précipité à $100 \%$ de saturation avec le sulfate d'ammonium. Le contenu en tyrosine a été déterminé par nous. Dans la littérature on trouve des chiffres oscillant entre $0,85 \% A$. $V$. Palladin) et 5,3\% (R. Block). 


\section{Sensibilité et exactitude de la nouvelle technique}

On peut doser avec le procédé décrit plus haut les protéines contenues dans des volumes de lait allant de $0 \mathrm{ml} .01$ à $0 \mathrm{ml} .2$ et contenant entre $0 \mathrm{mgr} .5$ et 5 milligrammes de matière protéique. Avec certaines modifications on pourrait aisément abaisser cette limite à 0 mgr. 1 .

Vérifiant l'exactitude de la nouvelle technique à l'aide de la méthode Kjeldahl, nous avons trouvé des différences qui ne dépassent pas $0,1 \%$.

\section{Conclusion}

1) Précisant les conditions de nitration et utilisant le photocolorimètre, on a résussi à accroître la sensibilité et l'exactitude du procédé de dosage des protéines du lait, proposé jadis par l'auteur.

2) Le domaine d'utilisation de cette nouvelle technique varie entre 0,5 et 5 milligrammes de protéines dans la prise d'essai. L'exactitude oscille autour de $0,1 \%$ en rapport avec la méthode de Kjeldahl.

3) L'extinction équivalente est presque égale pour les diverses protéines du lait, ce qui permet d'employer la même courbe étalon du photocolorimètre pour leurs dosages.

4) Il n'y a pas de relation entre le contenu en tyrosine des différentes protéines du lait et l'intensité de la réaction xanthoprotéique.

Lab. de Biochimie animale. Faculté de Médecine Vétérinaire ; Bucarest-Roumanie.

\section{BIBLIOGRAPHIE}

[1]Anibal Pinto Alṽarez et C. Medin. R. Rev. Quim. Farm. Santiago Ohili. 3, no 33, 6, 1945. Ref. Chim. Abst. 1946, 1239.

[2] L. M. Buruiana. Le Lait, 1933, T. XIII, No 130, 1214.

[3] L. M. Buruiana. Bul. Soc. Chimie Romînia. 1936, A. XVIII, n 3-4, 191.

[4] L. M. Buruiana. Arch. Vet. 1933, Vol. XXV, no 1-2, 55.

[5] R. Lefaux. Bull. Soc. Chim. Biol. 1935, T. XVII. No 12, 1822.

[6] Em. Riegler. Z. fûr Analyt. Chem. 1914, Bd. 53, 242.

[7] Fr. Russo. II Latte. 1958, 32, no 3, 167 